\title{
Clustering in free recall based upon input contiguity'
}

WILLIAM P. WALLACE, University of Nevada, Reno, Nev. 89507

A list of 16 words was exposed for one study trial in a modified free-recall experiment. Critical words in the list were paired randomly and presented three times each. For one group $(E)$ the two members of common pairs always appeared in successive positions during the study trial. For the other group (C) members of the predetermined random pairs were never presented successively. Clustering scores in recall based on the predetermined random pairings were significantly higher in Group $E$ than in Group C. It was concluded that adjacency relations during the study trial provided a sufficient basis for clustering during recall.

Associations among items in a list have been shown to provide a sufficient basis for clustering during free recall (e.g., Jenkins \& Russell, 1952). It has been demonstrated that associative strength among items can develop on the basis of the contiguous occurrence of those items (Keppel, 1966; Spear, Ekstrand, \& Underwood, 1964). The purpose of the present experiment was to determine if the contiguous occurrence of successive unrelated items during the study trial of a free-recall task would lead to clustering during recall. If associative strength develops between successive items during the study trial (input), and if associative strength produces clustering during free recall (output), then there ought to be a tendency for items which appeared adjacently during input to appear adjacently during a subsequent output trial.

The preceding argument leads to the expectation of considerable agreement between the order that items are presented on a study trial and the order in which they are recalled. Consistency between input order and output order has not been demonstrated with typical free-recall procedures (Asch \& Ebenholtz, 1962). However, with the following variations in the standard free-recall procedures, substantial consistency between input order and output order has been demonstrated: short list length which falls within the immediate memory span (Jahnke, 1965), list orders that approximate language structure (Deese \& Kaufman, 1957), and a relatively long free-study period of an entire list of items in a constant order. ${ }^{2}$. The present experiment involved a modification of the standard free-recall task that was designed to emphasize contiguity relations between pairs of words during a single study trial.

\section{MATERIALS}

A list of 16 unrelated words was presented for one study trial followed by a free-recall test. The list consisted of the 15 words from Deese's (1959) List 9 plus the inappropriate label. In constructing the list, four words were selected at random to serve as buffer words. Each buffer word appeared one time during the study trial. The buffer words were assigned to the first two and last two input positions in an effort to reduce the influence of primacy and recency effects on the recall of the remaining items. Each of the remaining 12 words was presented three times during input.

\section{PROCEDURE}

A modified free-recall procedure was utilized in presenting the 16-word list to two groups of Ss. The arrangement of words during input differed for the two groups. For the experimental (E) group, the 12 critical words were separated into six random pairs. The two members of a pair appeared in adjacent positions on all three presentations during input and in the same order within the pair. Each word was presented individually for $2 \mathrm{sec}$ by means of a Stowe memory drum, and a 2-sec blank interval followed the presentation of the second member of each pair. The order of the pairs within the list was determined randomly with the restriction that all pairs were presented $n$ times before any pair appeared $n+1$ times. Approximately 2 min were allowed for written recall.

For the control (C) group, each of the critical 12 words was assigned to three different pairs at random, such that no specific pair appeared more than once, and the specisic Group $\mathrm{E}$ pairings were not used. The presentation procedure was identical to that used for Group $E$ in all other respects. Thus, the adjacency relations for pairs of items were consistent on all three presentations during the study trial for Group E, but not for Group C.

\section{SUBJECTS}

A total of 40 introductory psychology students attending the University of Nevada participated in the experiment. The Ss were run individually, with $20 \mathrm{Ss}$ randomly assigned to each group.

RESULTS AND DISCUSSION
The question of major interest was whether clustering would occur on the predetermined basis of input contiguity. Clustering was assessed by using a modification of the ratio of repetition (Bousfield, Cohen, \& Whitmarsh, 1958). Bousfield et al subtracted 1 from the number of items recalled $(n)$ in their formula $(r / n-1)$ because the first item recalled cannot count as a repetition ( $r)$. In the present formulation $(r / n-k)$, $k$ was substracted from $n$ because the first item recalled from each of the $\mathbf{k}$ categories represented in recall cannot count as a repetition. Buffer items were not counted in n. Perfect clustering was indicated by a score of 1.00 and was independent of the number of items recalled.

Each of the random pairs defining the categories appeared three times for Group E and zero times for Group C. The mean clustering ratio based upon these categories was .432 for Group E and .193 for Group C. An arc-sin transformation was applied to the clustering scores to remove heterogeneity of variance. A one-way analysis of variance on the transformed clustering scores indicated that there was a significant difference between the groups $[F(1,38)=6.15, p<.05]$.

Group $\mathrm{C}$ was exposed to three different random pairings for each item during the study trial. Thus, there were three different sets of categories determined by the random pairings. The mean clustering ratio for Group $C$ based upon the first set of random pairs to appear in the list was .176, the mean clustering ratio based upon the second set of random pairs was .225 , and .346 for the third set. The clustering score for Group E was higher than each of these scores, although differences in category content make it difficult to evaluate such comparisons.

Buffer items appeared in the first two and last two positions during the study trial. It is possible that only two buffer items were not sufficient to eliminate the influence of a recency effect on the clustering scores. That is, if at the start of recall, Ss immediately wrote down the last four items viewed, clustering scores (based on the predetermined random pairings) would be enhanced for Group E, but not for Group C. The relatively high clustering ratios for Group C based on the third set of pairs they viewed may, in part, reflect such an advantage. Recomputing the Group E clustering ratios with the last critical pair of words excluded resulted in a slight increase in the mean clustering ratio (.433). When the same .random pair was excluded for Group $C$, they also showed an increase in the mean clustering ratio $(.242)$. However, the gains were modest, and it did not appear that a recency bias could account for all of the discrepancy in clustering scores between 
Table 2

Factor Loadings for the Slides

\begin{tabular}{crc}
\hline Slide Number & \multicolumn{3}{c}{ Factor } \\
\hline & \multicolumn{1}{c}{ A } & B \\
\hline 1 (c) & -42.715 & 42.724 \\
2 (n) & 62.185 & 31.766 \\
3 (c) & -40.480 & 37.511 \\
4 (n) & 75.830 & 20.066 \\
5 (n) & 76.101 & 22.861 \\
6 (c) & -55.697 & 32.407 \\
7 (c) & -57.045 & 26.368 \\
8 (n) & 79.649 & 22.056 \\
9 (n) & 75.997 & 23.008 \\
10 (c) & -62.421 & 34.184 \\
\hline
\end{tabular}

a congruous animal

$b$ incongruous animal

Table 3

Core Matrix for Subject Factor A

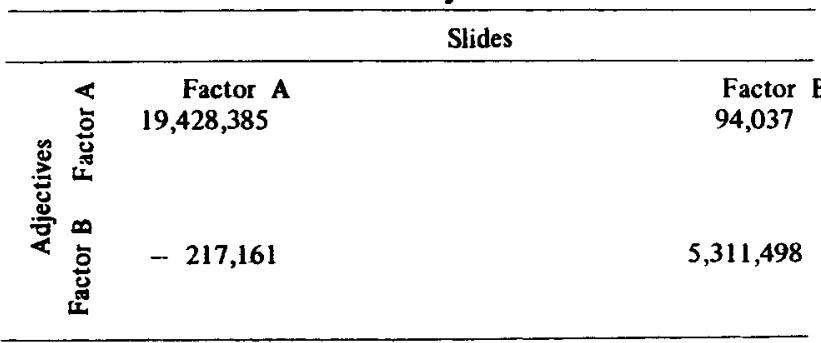

$66.5 \%$ and $14.7 \%$, respectively. The first factor could clearly be characterized as a congruity-incongruity dimension whereas the second factor again seemed to be a means factor. Thus, for stimuli and slides, novelty seems to be a unidimensional variable.

The results of the factor analysis also seemed to indicate that only one factor should be retained for Ss. The first factor accounted for $67 \%$ of the variance, while successive orthogonal axes each accounted for less than $5 \%$. Loadings on the first factor seemed to correspond to the mean judgments for an individual; hence, individual differences did not seem to reflect any large systematic deviation from the modal point of view.

The core matrix (Table 3 ) indicated that there was a very simple relationship between adjective and slide factors. The novelty factor for adjectives was highly related to the novelty factor for slides and the means factor for adjectives corresponded to the means factor for slides.

\section{REFERENCES}

BERLYNE, D. E. Conflict, arousal, and curiosity. New York: McGraw-Hill, 1960.

DEMBER, W. N. The psychology of perception. New York: Holt, Rinehart, \& Winston, 1960

FISKE, D. W., \& MADDI, S. R. A conceptual framework. In D. W. Fiske and S. R. Maddi (Eds.), Functions of varied experience. Homewood, Illinois: Dorsey, 1961. Pp. 11-56.

GLANZER, M. Curiosity, exploratory drive, and stimulus satiation. Psychological Bulletin, 1958, 55, 302-315.

TUCKER, L. R. The extension of factor analysis to three-dimensional matrices. In N. Frederiksen (Ed.), Contributions to mathematical psychology. New York: Holt, Rinehart, \& Winston, 1964.

WALSH, J. A. An IBM 709 program for factor analyzing three-mode matrices. Educational and Psychological Measurement, 1964, 24, 669-673.

NOTES

1. The authors wish to acknowledge both the Computation Center of the Massachusetts Institute of Technology where the data analysis was carried out on an IBM 7094 computer and the Tufts University Psychology Department which provided the necessary funding.

2. Now at Pennsylvania State University.

\section{(Continued from page 290)}

Group E and Group C. In examining the recall protocols it was noted that not one $S$ in Group $E$ included the clustered recall of the last critical pair among the first four items recalled.

There were six two-member categories defined by the random pairing used for Group E. Category recall was defined by the appearance of at least one member of the category during recall. The mean category recall for Group $E$ was 4.85 and for Group $C, 5.60$. The difference between groups was significant $[\mathrm{F}(1,38)=13.92, \mathrm{p}<.01]$.

Group $C$ surpassed Group $E$ in the number of words recalled. The mean recall for Group $C$ was 11.55 , compared to a mean recall of 10.40 for Group $E$. The difference between these means did not reach an acceptable level of statistical significance $[F(1,38)=3.31]$. The clustering advantage demonstrated in Group $\mathrm{E}$ was not accompanied by a similar advantage in correct recall.

There were very few outside-list intrusions or repetitions of list words during recall. The combined means of such responses per $\mathrm{S}$ were .35 for Group $\mathrm{E}$ and .45 for Group $\mathrm{C}$.

The present results demonstrated that input contiguity provided a sufficient basis for clustering during free recall. Similar results have been obtained by Greenhouse (1967), who found that temporal grouping of three and four unrelated words led to clustering of these words during recall. However, Greenhouse also reported advantages in number of words recalled following temporal grouping during presentation, a result which did not obtain in the present experiment.

\section{REFERENCES}

ASCH, S. E., \& EBENHOLTZ, S. M. The process of free recall: Evidence for non-associative factors in acquisition and retention. Journal of Psychology, 1962, 54, 3-31.

BOUSFIELD, W. A., COHEN, B. H., \& WHITMARSH, G. A. Associative clustering in the recall of words of different taxonomic frequencies of occurrence. Psychological Reports, 1958, 4, 39-44.

DEESE, J. Influence of inter-item associative strength upon immediate free recall. Psychological Reports, 1959, 5, 305-312.

DEESE, J., \& KAUFMAN, R. A. Serial effects in recall of unorganized and sequentially organized verbal material. Journal of Experimental Psychology, 1957, 54, 180-187.

GREENHOUSE, P. Free recall of categorized and unrelated words under serial or grouped presentation. Psychonomic Science, 1967, 8, 447448.

JAHNKE, J. C. Primacy and recency effects in serial-position curves of immediate recall. Journal of Experimental Psychology, 1965, 70, 130-132.

JENKINS, J. J., \& RUSSELL, W. A. Associative clustering during recall. Journal of Abnormal \& Social Psychology, 1952, 47, 818-821.

KEPPEL, G. Association by contiguity: Role of response availability. Journal of Experimental Psychology, 1966, 71, 624-628.

SPEAR, N. E., EKSTRAND, B. R., \& UNDERWOOD, B. J. Association by contiguity. Journal of Experimental Psychology, 1964, 67, 151-161.

\section{NOTES}

1. This research was supported by Grant GB8605 from the National Science Foundation.

2. Wallace, W. P. The influence of test trials on the development of subjective organization in free recall. Submitted for publication. 